\title{
Übersicht: Ergonomie
}

Zbl Arbeitsmed 2021 · 71:75-80 https://doi.org/10.1007/s40664-020-00408-4 Eingegangen: 20. Juli 2020

Angenommen: 15 . September 2020

Online publiziert: 7. Oktober 2020

(c) Der/die Autor(en) 2020

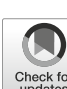

\section{Martin Braun}

Fraunhofer IAO - Fraunhofer-Institut für Arbeitswirtschaft und Organisation, Stuttgart, Deutschland

\section{Ausgangssituation der öffentlichen Verwaltung}

Die öffentliche Verwaltung ist der administrative Teil der staatlichen Exekutive, der öffentliche Aufgaben wahrnimmt. Sie versorgt Bürger und Unternehmen mit Verwaltungsleistungen; zudem schützt sie das Gemeinwohl und setzt das Recht um. Im föderalen System der Bundesrepublik Deutschland sind Bund, Länder und Kommunen die Träger der öffentlichen Verwaltung. Verwaltungsleistungen werden von unterschiedlichen Verwaltungsstellen und -ebenen erbracht. Handlungsträger der Verwaltung sind die Behörden. Zudem übernehmen ausgewählte Organisationen der Selbstverwaltung, wie Sozialversicherungen und Kammern, hoheitliche Verwaltungsaufgaben [14].

Aktueller Handlungsbedarf in der öffentlichen Verwaltung wurde im Rahmen des Modellprojekts „Digitale $\mathrm{Zu}$ kunftskommune@bw“ mittels Experteninterviews und Literaturrecherchen wie folgt erhoben:

\section{Personalkapazitäten}

Unter dem Zwang knapper Ressourcen hat die öffentliche Verwaltung verstärkt Personal abgebaut, zumal einige Behörden als überbesetzt galten. Die daraus resultierende Mehrbelastung des verbliebenen Personals beeinflusst die Qualität der Arbeit. Beschäftigte sehen sich zuweilen außerstande, das ihnen übertragene Arbeitspensum mit Sorgfalt zu erledigen. Demzufolge sind längere Bearbeitungs- zeiten, Fristversäumnisse und Regressansprüche nicht auszuschließen. Arbeitsbedingte Überforderung steigert die Unzufriedenheit der Beschäftigten und kann zu Stress, Erschöpfung und Befindensstörungen führen [11].

Unvorhersehbare Aufgabenstellungen - etwa im Zuge staatlicher Infektionsschutzkampagnen - überfordern die personellen und fachlichen Kapazitäten der Behörden. Zusatzaufgaben lassen sich nur durch eine Einbindung externer Assistenz bewältigen. Dennoch streben viele Behörden an, ihr Dienstleistungsangebot zu Zwecken der öffentlichen Daseinsfürsorge auszubauen.

\section{Entscheidungsinstanzen}

Als politische Vertretung der Bürgerschaft legt der Gemeinderat die Grundsätze für die kommunale Verwaltung fest und kontrolliert deren Einhaltung. Die Koordination von Gemeinderat und Kommunalverwaltung birgt ein strukturelles Konfliktpotenzial. Kommunalpolitiker konfrontieren die Verwaltungsbehörden zuweilen mit Aufgaben, die mit bestehenden Entscheidungsgängen und Arbeitsweisen kaum zu bewältigen sind. Unerfüllbare Aufträge und eine enge Kontrollpolitik des Gemeinderats begünstigen eine Planerfüllungslogik in der Verwaltung. Ein ergebnisorientiertes Verwaltungshandeln setzt indes voraus, dass sich die Politik auf strategische Zielvorgaben einigt und deren Umsetzung an die Verwaltung delegiert.

\section{Prozessorientierung}

Langwierige Entscheidungsprozesse erschweren es, behördliche Ressourcen in angemessener Zeit $\mathrm{zu}$ allokieren und zu aktivieren, um den Erwartungen der Verwaltungskunden gerecht $\mathrm{zu}$ werden. Folglich werden fallbezogene und prozessuale Verwaltungskonzepte angestrebt, die auf eine abteilungsübergreifende Aufgabenbewältigung mit kurzen Entscheidungsgängen fokussieren. Ein ausgeprägtes Ergebnis- und Verantwortungsbewusstsein der Beschäftigten soll sich positiv auf die Qualität und die Kosten von Verwaltungsleistungen auswirken. Allerdings ist die öffentliche Verwaltung an rechtliche Rahmenbedingungen gebunden, die einer rein betriebswirtschaftlichen Optimierungslogik entgegenstehen. Lassen sich die daraus resultierenden Widersprüche nicht integrieren, wirken sie sich nachteilig auf das Arbeitserleben der Beschäftigten aus.

\section{Verwaltungsmodernisierung}

Die öffentliche Verwaltung unterliegt einem anhaltenden Modernisierungsprozess, um die Kundenorientierung, das Qualitätsniveau und die Kosteneffizienz ihrer Leistungen ständig zu verbessern. Ziel- und ergebnisorientierte Steuerungsinstrumente des „Neuen Managements" sollen brachliegende Kreativitäts- und Produktivitätspotenziale erschließen. Erweiterte Entscheidungsund Handlungsspielräume ermutigen Beschäftigte zur aktiven Mitwirkung im 
Modernisierungsprozess und ermöglichen eine Entfaltung von Kenntnissen und Fertigkeiten im alltäglichen $\mathrm{Zu}$ sammenspiel von Mensch, Technik und Organisation [10].

Die bisherigen Erfolge der Verwaltungsmodernisierung genügen allerdings nicht allen Ansprüchen [9]. Beschäftigte erleben die Risiken der Modernisierung, wie Leistungsverdichtung, den Verlust von zwischenmenschlicher Verbindlichkeit und verringerte Sozialstandards. Ein sozialer Rückhalt im Kontext vertrauensvoller Informationsund Koordinationsprozesse erscheint unabdingbar, um erweiterte Tätigkeitsanforderungen zu erfüllen. Die notwendig zu intensivierenden Kommunikationsbeziehungen begründen dabei die hohen politischen Erwartungen an die Funktionspotenziale der digitalen Transformation in der öffentlichen Verwaltung.

\section{Vision der digitalen Transformation}

Die digitale Transformation steht für eine technologieinduzierte und -zentrierte Vision zur Vernetzung und Automatisierung von Arbeitssystemen. Ein Kernkonzept der digitalen Vernetzung von Arbeitsprozessen besteht darin, objektbezogene Informationen möglichst umfassend zu erheben, auszutauschen und auszuwerten. „Autonome Softwaresysteme" aufBasis künstlicher neuronaler Netze greifen auf massenhafte Datenbestände $\mathrm{zu}$, analysieren diese und extrahieren situationsspezifische Prozessinformationen. Durch eine Synchronisierung von virtuellen Datenmodellen und realen Arbeitsprozessen wird angestrebt, die Planung, Steuerung und Kontrolle von Verwaltungsprozessen $\mathrm{zu}$ verbessern [5].

Die Digitalisierung überträgt in zunehmendem Maße bislang dem Menschen vorbehaltene wissens- und denkgestützte Aufgaben an informationstechnische Maschinen. Digitale Informationssysteme können den arbeitenden Menschen überall dort entlasten oder gar substituieren, wo Algorithmen der Informationsverarbeitung vorliegen oder definierbare Heuristiken (d.h. Findehilfen) angegeben werden können [4].

\section{Digitalisierung öffentlicher Verwaltungsleistungen}

Im internationalen Vergleich des Digitalisierungsgrads von Verwaltungsleistungen belegt Deutschland hintere Ränge [8]. Entsprechend groß ist der Nachholbedarf bei der Umsetzung digitalisierter Verwaltungsangebote für Bürger und Unternehmen, für die der Gesetzgeber rechtliche Voraussetzungen geschaffen hat. Das Onlinezugangsgesetz (OZG) verpflichtet Bund, Länder und Kommunen, ihre Verwaltungsleistungen kurzfristig auch elektronisch über Verwaltungsportale anzubieten und diese zu einem Portalverbund fachlich zu verknüpfen. Den digitalisierten Angeboten liegen 575 Verwaltungsprozesse zugrunde, die 14 Themenfeldern zugeordnet sind. Das Onlinezugangsgesetz leitet einen Paradigmenwechsel ein, indem es die Nutzer in den Mittelpunkt des behördlichen Dienstleistungsangebots stellt [13].

Die digitale Transformation in der öffentlichen Verwaltung erweist sich als ein weitreichender Reorganisationsprozess, der auf eine konsequente Kundenorientierung und eine ressourcensparende Vereinfachung von Verwaltungsabläufen abzielt [5]. Überbehördliche Kooperationen, die auch regionale Unternehmen und Hochschulen einbeziehen können, tragen dazu bei, personelle und finanzielle Ressourcen zu bündeln und bewährte Lösungen zu transferieren. Die Digitalisierung von Verwaltungsleistungen eröffnet Möglichkeiten, die öffentlichen Daseinsfürsorge zu stärken und basisdemokratische Beteiligungsformen zu beleben. Zentrale Anwendungen werden nachfolgend skizziert.

\section{E-Government}

E-Government bezeichnet die Durchführung, Vereinfachung und Unterstützung von Prozessen zur Information, Kommunikation und Transaktion innerhalb und zwischen staatlichen, kommunalen und sonstigen Behörden sowie zwischen diesen Institutionen und Bürgern bzw. Unternehmen durch den Einsatz von digitalen Informationstechnologien. E-Government erweitert das behördliche Dienstleistungsangebot durch orts- und zeitunabhängige Zugangswege; so können Bürger etwa amtliche Urkunden von zuhause aus beantragen. Ein vereinfachter Zugang zu Informationsangeboten erhöht die Transparenz behördlichen Handelns, unterstützt den politischen Willensbildungsprozess und fördert die Bürgerbeteiligung [3].

\section{Elektronische Akte}

Das E-Government-Gesetz sieht eine sukzessive Umstellung der Aktenführung in der öffentlichen Verwaltung auf die elektronische Akte (E-Akte) vor. E-Akten sind standardisierte Lösungen auf Basis von Dokumentenmanagementsystemen. Sämtliche Dokumente, die die Verwaltung erreichen oder die diese erzeugt, werden in digitalisierter Form in elektronischen Archiven abgelegt. Sie sind somit schnell $\mathrm{zu}$ recherchieren. E-Akten sind von allen Berechtigten an jedem Ort und direkt aus einer Fachanwendung einzusehen.

\section{Datenplattformen}

Datenplattformen bzw. Verwaltungsportale unterstützen politische Entscheidungsprozesse, indem sie umfangreiche behördliche Datenbestände systematisch auswerten. Ihre soft- und hardwaretechnischen Komponenten ermöglichen es, Daten zu erfassen, zu analysieren und in anwendungsorientierte Ausgabeformate zu übertragen. Die Vorteile einer Datenplattform kommen allerdings nur bei einer intensiven Nutzerbeteiligung zum Tragen. Eine hohe Nutzerakzeptanz wiederum setzt eine vertrauenswürdige Systemarchitektur und ein hohes informatorisches Sicherheitsniveau voraus.

\section{Auswirkungen auf die menschliche Arbeit}

Die Digitalisierung verändert die Verwaltungsarbeit nachhaltig [3]. Im Alltag betrifft dies u. a. die Einführung der E-Akte, den Einsatz multimodaler Schnittstellengeräte oder die ergonomische Ausstattung mobiler Arbeitsplätze. Hier liegt eine Reihe bewährter Gestaltungsempfehlungen des Arbeitsschutzes vor [6]. Kernelemente der digitalen Transforma- 
tion beziehen sich allerdings weniger auf die Arbeitsmittel (d.h. Ebene der Objekte), sondern vielmehr auf die Organisation der Arbeit sowie eine zweckmäßige Funktionsteilung bzw. Interaktion von Mensch und Maschine (d.h. Ebenen der Struktur und der Prozesse). In $\bullet$ Tab. 1 werden differenzierte Kriterien der Verwaltungsmodernisierung benannt, woraus präventiver Handlungsbedarf abgeleitet wird.

Im Einzelfall können digitalisierte Arbeitsformen nicht nur die Handlungsoptionen der Beschäftigten erweitern, sondern auch deren arbeitsbezogene Gesundheitsgefahren vergrößern. Veränderte Berufsbilder und Arbeitstätigkeiten bergen das Risiko einer persönlichen Überforderung bzw. Verunsicherung, wenn etwa individuelle Lern- und Entwicklungsmöglichkeiten eingeschränkt werden oder sich die Transparenz von komplexen Entscheidungsgängen verliert. Damit derartige Einflüsse den Modernisierungsprozess in der öffentlichen Verwaltung nicht lähmen, müssen sich Entscheider vorsorglich mit Aspekten gesunder und sicherer Arbeit auseinandersetzen. Trotz erster, einschlägiger Erfahrungen ist den Entscheidern in den Verwaltungsbehörden allerdings nicht vollauf bewusst, in welchem Ausmaß die digitale Transformation die menschlichen Arbeitsbedingungen verändert und welche qualifikatorischen bzw. gesundheitlichen Wirkungen daraus für die Beschäftigten resultieren.

\section{Behördliche Erfahrungen}

Es erweist sich als dringlich, durch eine menschengerechte Gestaltung der Arbeit eine Antwort auf die mit dem Einsatz digitaler Technologien einhergehenden Veränderungen zu geben [12]. Pilothafte Erfahrungen bei der Digitalisierung in der öffentlichen Verwaltung sind wie folgt:

\section{Interne Hemmnisse}

Digitalisierungsprojekte stoßen auf diverse Widerstände, die u. a. auf fehlendes technisches Verständnis, mangelnde Weitsicht oder ausgeprägtes Konkurrenzverhalten zurückgehen. So ent-

Zbl Arbeitsmed 2021 · 71:75-80 https://doi.org/10.1007/s40664-020-00408-4

(c) Der/die Autor(en) 2020

\section{Braun \\ Impulse einer präventiven Arbeitsgestaltung zur Digitalisierung der öffentlichen Verwaltung}

\section{Zusammenfassung}

Um die Ansprüche von Politik und Bürgerschaft bestmöglich zu erfüllen, vollzieht die öffentliche Verwaltung einen anhaltenden Modernisierungsprozess. Die Steuerungsinstrumente des "Neuen Managements" sollen beitragen, menschliche Kreativitäts- und Produktivitätspotenziale zu aktivieren, um Verwaltungsleistungen kundenorientierter, qualitätsvoller und kosteneffizienter zu erbringen. Mittlerweile treiben die Funktionspotenziale des „E-Governments" die Verwaltungsmodernisierung weiter voran. Eine rechtliche Grundlage bildet das Onlinezugangsgesetz. Es verpflichtet die Behörden, ihre Verwaltungsleistungen auch mittels digitaler Verwaltungsportale anzubieten. Im Zuge der digitalen Transformation werden in zunehmendem Maße bislang dem Menschen vorbehaltene wissens- und denkgestützte Aufgaben auf Informationsmaschinen übertragen. Um zu vermeiden, dass die Reaktion auf unbefriedigende Arbeitsbedingungen den Modernisierungsprozess in der öffentlichen
Verwaltung lähmt, sind präventive Maßnahmen geboten. Diese erstrecken sich nicht nur auf den Einsatz von Arbeitsmitteln, sondern vor allem auf die Organisation der Arbeit sowie auf die Funktionsteilung von Mensch und Maschine. Der Beitrag stellt ausgewählte Handlungsfelder vor, die sich an einer "Kultur der Prävention" orientieren. Er zeigt auf, dass die digitale Transformation eine Aufwertung derjenigen menschlichen Leistungsbeiträge erfordert, die Maschinen nicht zu erbringen vermögen. Hierzu gehört vor allem die Ausgleichsfähigkeit des gesunden Menschen zur Förderung der sozialen Kohärenz. Kohärenz stellt hier ein Ordnungsmuster für komplexe soziotechnische Arbeitssysteme dar, die sich den Regeln des rationalen Planungsparadigmas entziehen.

\section{Schlüsselwörter}

Arbeitsgestaltung · Prävention · Humanzentrierung · Digitalisierung · Öffentliche Verwaltung

\section{Impulses of a preventive work design in the digitalization of public administration}

\section{Abstract}

In order to successfully fulfil the demands of politics and the citizenry, the public administration is undergoing a continuous modernization process. The instruments of the new management are intended to activate the human creativity and productivity potentials in order to provide more customer-oriented administrative services, of high quality and more cost efficiently. In the meantime, the functional potential of the e-government is driving the modernization of administration further forward. A legal foundation is the Online Access Act, which obliges public authorities to provide their administrative services via digital portals. In the course of the digital transformation, knowledge-based and thinkbased tasks previously reserved for humans are increasingly being transferred to information machines. In order to avoid that the reaction to unsatisfactory working conditions paralyses the modernization process in the public administration, preventive measures are recommended. Prevention covers not only the use of work equipment but above all the organization of work and the division of functions between man and machine. This article presents selected fields of action that are oriented towards a culture of prevention. It shows that digital transformation requires an upgrading of those human performances that machines are not able to provide. This includes, above all, the balancing ability of healthy people to promote social coherence. Coherence represents an ordering pattern for complex sociotechnical working systems that evade the rules of the rational planning paradigm.

\section{Keywords}

Work design · Prevention · Human centered design · Digitalization · Public administration 
Tab. 1 Kriterien der Verwaltungsmodernisierung und präventiver Handlungsbedarf. (Eigene Darstellung)

\begin{tabular}{|c|c|c|c|}
\hline Betrachtungsebene & Kriterien tradierter Verwaltung & Kriterien digitaler Verwaltung & Präventiver Handlungsbedarf \\
\hline \multirow[t]{4}{*}{ Paradigmen } & Bürokratie & Kundenorientierung & Humanzentrierte Vision und Werte \\
\hline & Zentralismus & Projektorganisation & Integrativer Gestaltungsansatz \\
\hline & \multirow[t]{2}{*}{ Abgegrenzte Zuständigkeiten } & Lebenslagenbezogene Angebote & \multirow[t]{2}{*}{ Ethische Kriterien der Technisierung } \\
\hline & & Dezentrale Portalverbünde & \\
\hline \multirow[t]{5}{*}{ Mentale Konzepte } & Rationale Systemsicht & Integrative Systemsicht & Stärkung der behördlichen Identität \\
\hline & Vorschriftenorientierung & Bedarfsorientierung & $\begin{array}{l}\text { Umgang mit Quantitäten und Qua- } \\
\text { litäten }\end{array}$ \\
\hline & „Silodenken” & Mission der Nachhaltigkeit & \multirow[t]{3}{*}{ Förderung der Resilienz } \\
\hline & \multirow[t]{2}{*}{ Exklusive Logik („entweder-oder") } & Interessensausgleich & \\
\hline & & Inklusive Logik („,sowohl-als-auch“) & \\
\hline \multirow{4}{*}{$\begin{array}{l}\text { Strukturen, } \\
\text { Rahmenbedingungen }\end{array}$} & Stationäres Systemverhalten & Dynamisches Systemverhalten & Kohärente Ordnungsmuster \\
\hline & Hierarchische Aufbauorganisation & Netzwerkstrukturen & Arbeits- und Funktionsteilung \\
\hline & \multirow[t]{2}{*}{ Top-down-Struktur } & \multirow[t]{2}{*}{ Horizontale und vertikale Kooperation } & Vertrauensvolle Kooperation \\
\hline & & & $\begin{array}{l}\text { Problemlösungs-orientierte Partizi- } \\
\text { pation }\end{array}$ \\
\hline \multirow[t]{3}{*}{ Prozesse } & Formalisierte Geschäftsgänge & Ungeplante Geschäftsgänge & Kapazitäts- und Zeitbemessung \\
\hline & \multirow[t]{2}{*}{ Reaktivität } & Kontinuierliche Verbesserung & Lernförderliche Arbeit \\
\hline & & Freiraum für „Experimente" & Störungsmanagement \\
\hline \multirow[t]{3}{*}{ Objekte, Ereignisse } & $\begin{array}{l}\text { Arbeitsmittel mit geringer digitaler } \\
\text { Durchdringung }\end{array}$ & $\begin{array}{l}\text { Vernetzte Informations- und Kooperati- } \\
\text { onssysteme }\end{array}$ & Datenschutz und -sicherheit \\
\hline & \multirow[t]{2}{*}{ Vielfalt der Medien und ihrer Formate } & Schnittstellen- und Dialogsysteme & Usability, User Experience \\
\hline & & Standardisierter Datenaustausch & $\begin{array}{l}\text { Ergonomische Arbeitsplatzgestal- } \\
\text { tung }\end{array}$ \\
\hline
\end{tabular}

spricht etwa die standardisierte Kommunikation über einen Portalverbund nicht den gewachsenen Strukturen und Zuständigkeiten der öffentlichen Verwaltung. Sie läuft auch den gewohnten Handlungsweisen der Beteiligten zuwider. Werden bewährte Routinen aufgegeben oder verschieben sich institutionelle Machtverhältnisse, so kann dies zu Ängsten und Abwehrhaltungen der Beschäftigten führen, welche eine Projektumsetzung erschweren.

\section{Unzureichende Gestaltungs- expertise}

In den Behörden sind üblicherweise keine professionellen Arbeitsgestalter tätig, die den Prozess der Digitalisierung fachkundig begleiten könnten. Zudem liegen zumeist keine systematischen Vorgehensweisen und Instrumente zur Umsetzung von Modernisierungsprojekten vor. Arbeitsgestaltung ist vielmehr ein Bestandteil des alltäglichen Handelns der höheren Führungskräfte, wie Bürgermeister und Amtsleiter. Sie schaffen personelle und sachliche Voraussetzun- gen u.a. für die Gestaltung technischorganisatorischer Arbeitsbedingungen, die Personalrekrutierung und die Ausund Weiterbildung von Beschäftigten. Zur Ausgestaltung des digitalen Wandels bedürfen die Führungskräfte indes weitergehender fachlicher Unterstützung.

\section{Externe Unterstützung}

Als Träger der gesetzlichen Unfallversicherung stehen die Unfallkassen in engem Kontakt mit den Behörden. Sie begleiten den Prozess der digitalen Transformation in der öffentlichen Verwaltung fachkundig und unterstützen die Akteure im Rahmen ihres Präventionsauftrags [6]. Einschlägige Erfahrungen offenbaren allerdings, dass weitergehender Aufklärungs- und Informationsbedarf über die sachlogischen Zusammenhänge von gesundheitlicher Prävention und digitaler Transformation besteht. Neben praktischen Hinweisen zur Maßnahmenumsetzung gilt es, die Nutzenpotenziale einer menschengerechten Arbeitsgestaltung aufzuzeigen.

\section{Handlungsfelder der Prävention}

Die Arbeitsgestaltung widmet sich der optimalen Gestaltung von Arbeitssystemen, -prozessen und -bedingungen. Prävention bezeichnet in diesem $\mathrm{Zu}$ sammenhang eine gedankliche Vorwegnahme möglicher körperlicher bzw. psychischer Schädigungen sowie von Beeinträchtigungen des gesundheitlichen Befindens. Sie folgt arbeitswissenschaftlichen Konzepten bereits im Stadium des Entwurfs von Arbeitssystemen und -prozessen. Im Kontext der Digitalisierung der öffentlichen Verwaltung werden nachfolgend sieben präventive Handlungsfelder vorgestellt. Sie orientieren sich konzeptionell an der „Kultur der Prävention“, die im Mittelpunkt der Präventionskampagne „kommitmensch“ der Deutschen Gesetzlichen Unfallversicherung (DGUV) steht [2].

\section{Handlungsfeld Führung}

In komplex vernetzten Arbeitssystemen kommt der Organisation von Widersprüchlichkeit und Instabilität eine er- 
folgskritische Bedeutung zu. Der professionelle Umgang mit Dynamik und die Fähigkeit, Fach- und Entscheidungskompetenzen aufgabengerecht zu bündeln, prägt die Wirksamkeit von Verwaltungsprozessen. Der Führung kommt dabei die Aufgabe zu, für eine situationsgerechte Balance von Vielfalt und Ordnung im Arbeitsbereich zu sorgen. Zur Integration dieser Antagonismen existieren keine vorgefertigten Lösungen. Vielmehr hat Führung einen verbindlichen Handlungsrahmen zu schaffen, indem sie für eine Transparenz von Leistungsbeiträgen sorgt, divergierende Interessenslagen der Beteiligten koordiniert und taktische Spiele aufdeckt. Ferner hält ihre glaubwürdige Vorbildrolle die organisationale Veränderungsbereitschaft wach.

\section{Handlungsfeld Kommunikation}

Der ursprüngliche Wortsinn von „Kommunikation “ beschreibt eine Sozialhandlung. Kommunikation dient dem Wissensaustausch, der Abstimmung und der Entscheidung hinsichtlich Veränderung und Problemlösung. Hierzu bezieht sie gleichermaßen Beratung, Anleitung und Rückmeldung ein. Soweit sich in der Kommunikation ein wechselseitiges Geben und Nehmen von Wissen manifestiert, bedürfen ihre Prozesse einer kulturellen Komponente des sozialen Ausgleichs. Aufgabe der Fachund Führungskräfte ist es, die zwischenmenschlichen Kommunikationsund Austauschprozesse in verbindlicher Weise zu moderieren.

\section{Handlungsfeld Beteiligung}

Organisationen verwirklichen die Idee einer zielgerichteten Kooperation, indem sie die vielfältigen Fähigkeiten und Kenntnisse ihrer Beschäftigten, insbesondere ihre erfahrungsgeleitete Urteils- und Entscheidungsfähigkeit, bündeln und auf konkrete Aufgaben anwenden. Um ihre Koordinationskapazitäten nicht zu überfordern, richtet eine beteiligungsorientierte Organisation ihre Entscheidungsstrukturen am verfügbaren Problemlösungswissen aus. Problemlösungsorientierte Partizipation setzt ein verantwortungsvolles Rollen- verständnis aller Beteiligten voraus. So können erfahrene Arbeitspersonen, die aufgrund ihrer Expertise eine fachliche Reputation und soziale Anerkennung erworben haben, fachliche Entscheidungsfunktionen übernehmen. Weitere Merkmale beteiligungsorientierter Strukturen sind die Zugänglichkeit von Informationsbeständen, die Transparenz von Entscheidungsprozessen und allgemein akzeptierte Wege zu gerecht empfundenen Konfliktlösungen. Indem Beschäftigte in ihrem Delegationsbereich zur Entscheidungsfindung beitragen, wachsen ihre Identifikation mit der Arbeitstätigkeit und ihre Loyalität zum sozialen Arbeitsumfeld.

\section{Handlungsfeld Fehlerkultur}

Das rationale Paradigma betrachtet Arbeitssysteme als quantifizier- und analysierbare Elemente mit präzise planbaren Ursache-Wirkungs-Beziehungen. Angesichts dynamisch veränderlicher oder gar widersprüchlicher Arbeitsverhältnisse fällt es zunehmend schwer, die Ziele und Mittel von Arbeit hinreichend genau zu operationalisieren und zu steuern [8]. Sofern es immer weniger gelingt, quantitative Planabweichungen zu bestimmen, ist eine situative Fehlerkultur geboten. Hierbei wird ein Misslingen weniger als ein Fehler, sondern als ein "gescheiterter Versuch" betrachtet. Ein solches Scheitern birgt etwas Positives, wenn sich Beschäftigte zu ihren Fehlhandlungen bekennen und daraus lernen. Unterliegen Beschäftigte allerdings einem Rechtfertigungszwang, so sehen sie keinen Anlass, ihr Misslingen zu reflektieren. Eine konstruktive Fehlerkultur setzt demnach lernförderliche Verhältnisse voraus, in denen Beschäftigte situationsgerecht aus Einsicht und in freier Verantwortung handeln.

\section{Handlungsfeld Arbeitsklima}

Neben objektiven Kriterien der Auftragserfüllung erweisen sich subjektive Dimensionen des Arbeitsklimas als erfolgsrelevante Elemente behördlichen Handelns. Arbeitsklima ist die subjektiv erlebte, längerfristige Qualität der menschlichen Zusammenarbeit, die sich auf Ar- beitsmotivation und Arbeitserfolg auswirkt. Das Arbeitsklima wird maßgebend durch die Behördenkultur geprägt. Diese kennzeichnen den Umgang, das Auftreten und Benehmen von Beschäftigten und Führungskräften untereinander sowie gegenüber externen Anspruchsgruppen. Angestrebt wird eine zielorientierte, loyale und verträgliche Behördenkultur. Dieser liegt idealerweise ein Wertesystem zugrunde, das Chancenvielfalt, Vertrauen und Verantwortung verwirklicht - und hierdurch Orientierung bei (konflikthaften) Entscheidungen vermittelt.

\section{Handlungsfeld Sicherheit}

Sicherheit ist der Ausgangspunkt einer produktiven und zuverlässigen Arbeitsweise. In digital vernetzten Arbeitssystemen umfasst sie - neben der etablierten Arbeitssicherheit und der Sicherheitstechnik - vor allem die informationstechnische Sicherheit (d.h. Internet Security) und den Datenschutz (d.h. Internet Safety). Mit fortschreitendem Digitalisierungsgrad nehmen die Sicherheitsrisiken zu. Firewalls, Anti-Viren-Programme und „IntrusionDetection"-Systeme reichen für eine Abwehr von Cyberkriminalität nicht mehr aus. Letztlich sind informationstechnische Sicherheitsrisiken vor allem durch umsichtige und loyale Verhaltensweisen der Beschäftigten zu minimieren.

\section{Handlungsfeld Gesundheit}

Das organisatorische Konzept der Arbeitsteilung impliziert eine Reihe struktureller Antagonismen, so etwa im Verhältnis von Spezialisierung und Koordination oder im Verhältnis von Nachfrage und Angebot. Die Systemtheorie definiert Gesundheit als eine regulative Fähigkeit, um die komplementären Pole derartiger Spannungsfelder zu integrieren. Konzeptionelle Grundlage dieses erweiterten Gesundheitsverständnisses ist die inklusive Logik des "Sowohlals-auch" [1]. Die Beherrschung der inklusiven Logik ist dem mündigen Menschen vorbehalten, während sich die digitale Maschinenlogik mit ihren binären Signalen bei aller mathematischen Präzision auf das exklusive Prinzip des „Ent- 
weder-oder" beschränkt [7]. Diese konzeptionelle Perspektive begründet den unabdingbaren Stellenwert des gesunden Menschen im soziotechnischen Arbeitssystem. Unter veränderlichen, begrenzt prognostizierbaren Umweltbedingungen kommt ihm die anspruchsvolle Aufgabe zu, sowohl die Identität eines Arbeitssystems zu stärken, als auch dessen Anpassungs- und Anschlussfähigkeit zu fördern.

\section{Ausblick}

Mit der Digitalisierung von behördlichen Leistungsprozessen wird ein technozentrischer Ansatz zur Modernisierung der öffentlichen Verwaltung verfolgt. Einschlägige Erfahrungen offenbaren, dass die digitale Transformation eine Aufwertung unabdingbarer menschlicher Leistungen erfordert, welche Maschinen nicht zu erbringen vermögen. Hierzu gehört vor allem die Ausgleichsfähigkeit des gesunden Menschen zur Förderung der sozialen Kohärenz [1]. Kohärente Ordnungsmuster erweisen sich als eine Voraussetzung zur Beherrschung begrenzt prognostizierbarer Verhaltensweisen komplexer Systeme, die sich den Regeln des rationalen Planungsparadigmas entziehen [7]. Innerhalb einer humanzentrierten Modernisierungsstrategie sorgt ein präventiver Gestaltungsansatz für die notwendigen Impulse und Freiräume, um kohärente Strukturen aus dem Arbeitssystem selbst heraus zu entwickeln.

\section{Fazit für die Praxis}

- Die öffentliche Verwaltung befindet sich in einem Modernisierungsprozess; dieser zielt darauf, Verwaltungsleistungen kundenorientierter und kosteneffizienter zu erbringen.

- Um die Modernisierungseffekte zu verstärken, soll die Verwaltungsmodernisierung durch das E-Government weiter vorangetrieben werden.

- Im Zuge der Digitalisierung werden bislang dem Menschen vorbehaltene, denkgestützte Aufgaben zunehmend auf Informationsmaschinen übertragen.
- Die Reaktion auf unangemessene Arbeitsbedingungen kann den Modernisierungsprozess lähmen.

- Folglich sind präventive Maßnahmen der Arbeitsgestaltung geboten, die unabdingbare menschliche Leistungsbeiträge im Verwaltungsprozess aufwerten.

- Die Ausgleichsfähigkeit des gesunden Menschen erweist sich als eine Voraussetzung für die Beherrschung einer begrenzt prognostizierbaren Systemdynamik.

- Der vorliegende Beitrag stellt Handlungsfelder vor, die sich an einer „Kultur der Prävention“ orientieren.

\section{Korrespondenzadresse}

Dr. Martin Braun

Fraunhofer IAO - Fraunhofer-Institut für

Arbeitswirtschaft und Organisation

Nobelstraße 12, 70569 Stuttgart, Deutschland martin.braun@iao.fraunhofer.de

Funding. Open Access funding enabled and organized by Projekt DEAL.

\section{Einhaltung ethischer Richtlinien}

Interessenkonflikt. Der Artikel beruht auf ausgewählten Ergebnissen eines Forschungsanwendungsprojekts des Fraunhofer IAO im Auftrag der Unfallkasse Baden-Württemberg. M. Braun gibt an, dass kein Interessenkonflikt besteht.

Für diesen Beitrag wurden vom Autor keine Studien an Menschen oder Tieren durchgeführt. Für die aufgeführten Studien gelten die jeweils dort angegebenen ethischen Richtlinien.

Open Access. Dieser Artikel wird unter der Creative Commons Namensnennung 4.0 International Lizenz veröffentlicht, welche die Nutzung, Vervielfältigung, Bearbeitung, Verbreitung und Wiedergabe in jeglichem Medium und Format erlaubt, sofern Sie den/die ursprünglichen Autor(en) und die Quelle ordnungsgemäß nennen, einen Link zur Creative Commons Lizenz beifügen und angeben, ob Änderungen vorgenommen wurden.

Die in diesem Artikel enthaltenen Bilder und sonstiges Drittmaterial unterliegen ebenfalls der genannten Creative Commons Lizenz, sofern sich aus der Abbildungslegende nichts anderes ergibt. Sofern das betreffende Material nicht unter der genannten Creative Commons Lizenz steht und die betreffende Handlung nicht nach gesetzlichen Vorschriften erlaubt ist, ist für die oben aufgeführten Weiterverwendungen des Materials die Einwilligung des jeweiligen Rechteinhabers einzuholen.
Weitere Details zur Lizenz entnehmen Sie bitte der Lizenzinformation auf http://creativecommons.org/ licenses/by/4.0/deed.de.

\section{Literatur}

1. Braun M (2017) Arbeit 4.0: Der gesunde Mensch in der digitalisierten Arbeitswelt. In:NowakD, Letzel S (Hrsg) Handbuch der Arbeitsmedizin. Ecomed, Landsberg, S1-24 (4. Ergänzungslieferung)

2. Deutsche Gesetzliche Unfallversicherung - DGUV (2017) Fachkonzept für die nächste gemeinsame Präventionskampagne der DGUV und ihrer Mitglieder.DGUV, Berlin

3. Etscheid J, von Lücke J, Stroh F (2020) Künstliche Intelligenz in der öffentlichen Verwaltung. Anwendungsfelder und Szenarien. Fraunhofer, Stuttgart

4. Hacker W (2018) Menschengerechtes Arbeiten in der digitalisierten Welt. vdf, Zürich

5. Heuermann R, Tomenendal M, Bressem C (2018) Digitalisierung in Bund, Ländern und Gemeinden: ITOrganisation, Management und Empfehlungen. Springer Gabler, Wiesbaden

6. Hoffmann K (2019) Digitalisierung in der öffentlichen Verwaltung sicher und gesund gestalten. DGUVForum 12:18-19

7. Huchler N (2018) Grenzen der Digitalisierung von Arbeit - die Nicht-Digitalisierbarkeit und Notwendigkeit impliziten Erfahrungswissens und informellen Handelns. Z Arbeitswiss 72:215-223

8. Kirchhof R (2003) Ganzheitliches Komplexitätsmanagement: Grundlagen und Methodik des Umgangs mit Komplexität im Unternehmen. Deutscher Universitäts-Verlag, Wiesbaden

9. Krcmar H, Dapp M, Zepic R, Müller L, Dietrich S, Boberach M, Moy T (2016) E-Government Monitor 2016. Nutzung und Akzeptanz digitaler Verwaltungsangebote - Deutschland, Österreich und Schweiz im Vergleich. Initiative D21, Berlin

10. Naschold F (1995) Modernisierung des öffentlichen Sektors in Europa. Ergebnissteuerung, Wettbewerb und gesellschaftliche Devolution. In: Behrends F, Heinze RG, Hilbert J, Stöbe S (Hrsg) Der neue Start. Sigma, Berlin, S $31 \mathrm{ff}$

11. Oppen M (2011) Dienstleistungsarbeit unter öffentlich-privater Regie - größere Chancen und mehrRisiken. WZBrief Arbeit 10:6

12. Rothe I, Wischniewski S, Tegtmeier $P$, Tisch $A$ (2019) Arbeiten in der digitalen Transformation Chancen und Risiken für die menschengerechte Arbeitsgestaltung. Z Arbeitswiss 73:246-251

13. Stocksmeier D, Hunnius S (2018) OZG-Umsetzungskatalog. Digitale Verwaltungsleistungen im Sinne des Onlinezugangsgesetzes. Bundesministerium des Innern, für Bau und Heimat, Berlin

14. Strunz H (1993) Der Verwaltungsbegriff. In: Hofmann M, Zapotoczky K, Strunz H (Hrsg) Gestaltung öffentlicher Verwaltungen. Springer, Berlin, S3-35 